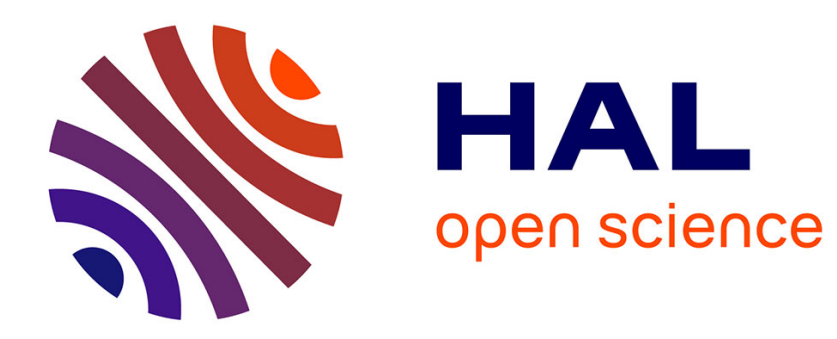

\title{
The bideterministic concatenation product
}

Jean-Eric Pin, Denis Thérien

\section{- To cite this version:}

Jean-Eric Pin, Denis Thérien. The bideterministic concatenation product. International Journal of Algebra and Computation, 1993, 3, pp.535-555. hal-00020072

\section{HAL Id: hal-00020072 \\ https://hal.science/hal-00020072}

Submitted on 4 Mar 2006

HAL is a multi-disciplinary open access archive for the deposit and dissemination of scientific research documents, whether they are published or not. The documents may come from teaching and research institutions in France or abroad, or from public or private research centers.
L'archive ouverte pluridisciplinaire $\mathbf{H A L}$, est destinée au dépôt et à la diffusion de documents scientifiques de niveau recherche, publiés ou non, émanant des établissements d'enseignement et de recherche français ou étrangers, des laboratoires publics ou privés. 


\title{
The bideterministic concatenation product
}

\author{
Jean-Eric Pin and Denis Thérien* \\ Bull Research and Development, Rue Jean-Jaurès, 78340 Les \\ Clayes-sous-Bois, France
}

\begin{abstract}
This paper is devoted to the study of the bideterministic concatenation product, a variant of the concatenation product. We give an algebraic characterization of the varieties of languages closed under this product. More precisely, let $\mathbf{V}$ be a variety of monoids, $\mathcal{V}$ the corresponding variety of languages and $\hat{\mathcal{V}}$ the smallest variety containing $\mathcal{V}$ and the bideterministic products of two languages of $\mathcal{V}$. We give an algebraic description of the variety of monoids $\widehat{\mathbf{V}}$ corresponding to $\hat{\mathcal{V}}$. For instance, we compute $\widehat{\mathbf{V}}$ when $\mathbf{V}$ is one of the following varieties : the variety of idempotent and commutative monoids, the variety of monoids which are semilattices of groups of a given variety of groups, the variety of $\mathcal{R}$-trivial and idempotent monoids. In particular, we show that the smallest variety of languages closed under bideterministic product and containing the language $\{1\}$, corresponds to the variety of $\mathcal{J}$-trivial monoids with commuting idempotents. Similar results were known for the other variants of the concatenation product, but the corresponding algebraic operations on varieties of monoids were based on variants of the semidirect product and of the Malcev product. Here the operation $\mathbf{V} \rightarrow \widehat{\mathbf{V}}$ makes use of a construction which associates to any finite monoid $M$ an expansion $\widehat{M}$, with the following properties:
\end{abstract}

(1) $M$ is a quotient of $\widehat{M}$,

(2) the morphism $\pi: \widehat{M} \rightarrow M$ induces an isomorphism between the submonoids of $\widehat{M}$ and of $M$ generated by the regular elements and

(3) the inverse image under $\pi$ of an idempotent of $M$ is a 2-nilpotent semigroup.

This paper assumes some familiarity with Eilenberg's theory of varieties and especially the notion of syntactic monoid of a recognizable language. References for this theory are $[6,7,10]$. The main result of this theory states that there exists a one-to-one correspondence between certain families of recognizable sets (the varieties of languages) and certain families of finite semigroups (the varieties of finite semigroups).

A fundamental result of Schützenberger [13] states that the smallest variety of languages closed under concatenation product corresponds to the variety of aperiodic monoids. Since then, an important part of the existing literature on varieties has been devoted to the study of the concatenation product and its variants. These variants include the weak forms of the concatenation product introduced by Schützenberger [14] (the unambiguous product and the left and right deterministic products) and the counter product introduced by Straubing [15]. This paper is devoted to the study of the two-sided version of the deterministic products, called the bideterministic product.

\footnotetext{
* Research on this paper was supported for the first author by PRC "Mathématiques et Informatique" and for the second author by the NSERC grant no. A4546 et FCAR grant no 89-EQ-2933.
} 
The general setting for this type of result can be summarized as follows. Let o be a binary operation on languages - in our case the concatenation product or one of its variants - and let $\mathcal{V}$ be the variety of languages corresponding to a variety of monoids $\mathbf{V}$. Denote by $\mathcal{V}^{\prime}$ the smallest variety containing $\mathcal{V}$ and closed under $\circ$. The question is to describe the varieties of monoids $\mathbf{V}^{\prime}$ corresponding to $\mathcal{V}^{\prime}$. For all the variants of concatenation mentionned previously, the variety $\mathbf{V}^{\prime}$ is equal to a Malcev product of the form $\mathbf{W} \mathbb{M} \mathbf{V}$, where $\mathbf{W}$ is a certain variety of semigroups $[16,8,9,12,17,18]$. This variety $\mathbf{W}$ is given in the following table:

\begin{tabular}{|l|l|}
\hline Product type & Variety $\mathbf{W}$ such that $\mathbf{V}^{\prime}=\mathbf{W}$ (i) $\mathbf{V}$ \\
\hline concatenation & aperiodic semigroups \\
\hline unambiguous & semigroups $S$ such that $e S e=e$ for each idempotent $e \in S$ \\
\hline right deterministic & semigroups $S$ such that $e S=e$ for each idempotent $e \in S$ \\
\hline left deterministic & semigroups $S$ such that $S e=e$ for each idempotent $e \in S$ \\
\hline counter & semigroups which are locally solvable groups \\
\hline
\end{tabular}

This is no longer true for the bideterministic product: in this case, the variety $\mathbf{V}^{\prime}$ cannot be written as a Malcev product of some variety with $\mathbf{V}$ and a new algebraic operation is required. This new operation relies on a construction of independent interest, which associates to any monoid $M$ a certain expansion $\widehat{M}$, with the following properties: $M$ is a quotient of $\widehat{M}$ and the morphism $\pi: \widehat{M} \rightarrow M$ induces an isomorphism from $\langle\operatorname{Reg}(\widehat{M})\rangle$, the submonoid of $\widehat{M}$ generated by the regular elements of $\widehat{M}$, onto $\langle\operatorname{Reg}(M)\rangle$. Furthermore, the inverse image under $\pi$ of an idempotent of $M$ is a 2-nilpotent semigroup. Our construction is somewhat reminiscent of the expansion proposed by Birget, Margolis and Rhodes in $[4,5]$, but turns out to be different, as we shall see on an example.

Now the key result states that a variety of languages is closed under bideterministic product if and only if the corresponding variety of monoids is closed under this expansion. We also give a more precise version of this result. Let $\mathcal{V}$ be a variety of languages and let $\mathbf{V}$ be the corresponding variety of monoids. Let $\hat{\mathcal{V}}$ be the smallest variety containing $\mathcal{V}$ and the bideterministic products of two languages of $\mathcal{V}$. Then the variety of monoids corresponding to $\hat{\mathcal{V}}$ is the variety of monoids generated by the monoids of the form $\hat{M}$ for some $M \in \mathbf{V}$. Similar results are known for the other variants of products, but again, there are based on totally different algebraic constructions (essentially variants of the semidirect product).

We compute $\widehat{\mathbf{V}}$ for various varities $\mathbf{V}$, including the variety of idempotent and commutative monoids, the variety of monoids which are semilattices of groups of a given variety of groups and the variety of $\mathcal{R}$-trivial and idempotent monoids.

As a byproduct, we characterize the smallest non trivial variety of languages containing the language $\{1\}$ and closed under bideterministic product : the corresponding variety of monoids is the variety of $\mathcal{J}$-trivial monoids whose idempotents commute.

\section{Some preliminaries.}

In this section, we recall some basic definitions or facts about finite semigroups and languages. All semigroups and monoids considered in this paper are either finite or free, although some results could be easily extended to periodic semigroups.

Let $S$ be a semigroup. We denote by $S^{1}$ the semigroup equal to $S$ if $S$ has an identity and to $S \cup\{1\}$, where 1 is a new identity, otherwise. We denote by $\mathrm{E}(S)$ the set of idempotents of $S$. For each element $s$ of $S$, the subsemigroup of $S$ generated 
by $s$ contains a unique idempotent, denoted $s^{\omega}$. If $P$ is a subset of $M,\langle P\rangle$ denotes the submonoid generated by $P$.

Given $s, t \in S$, we say that $s$ is $\mathcal{R}$-below $t$ (denoted $s \leq_{\mathcal{R}} t$ ) if there exists $x \in S^{1}$ such that $s=t x$. The elements $s$ and $t$ are $\mathcal{R}$-equivalent (denoted $s \mathcal{R} t$ ) if $s \leq_{\mathcal{R}} t$ and $t \leq_{\mathcal{R}} s$. Finally, we denote $s<_{\mathcal{R}} t$ if $s$ is $\mathcal{R}$-below $t$ but is not $\mathcal{R}$-equivalent with $t$. The relations $\leq_{\mathcal{L}}, \mathcal{L}$ and $<_{\mathcal{L}}$ are defined dually. For instance, $s \leq_{\mathcal{L}} t$ if there exists $x \in S^{1}$ such that $s=x t$.

Let $s$ be a semigroup and let $s$ be an element of $S$. An element $\bar{s}$ of $S$ is called a weak inverse of $s$ if $\bar{s} s \bar{s}=\bar{s}$. It is an inverse of $s$ if $\bar{s} s \bar{s}=\bar{s}$ and $s \bar{s} s=s$. In this case, $s$ is an inverse of $\bar{s}$. An element which has an inverse is called regular. We denote by $\operatorname{Reg}(S)$ the set of regular elements of a semigroup $S$. The following propositions state some elementary properties of weak inverses.

Proposition 1.1 Let $\bar{s}$ be a weak inverse of $s$. Then $s \bar{s}$ and $\bar{s} s$ are idempotent and $\bar{s}$ is an inverse of $s \bar{s} s$.

Proof. If $\bar{s}$ is a weak inverse of $s$, we have $\bar{s} s \bar{s}=\bar{s}$. This implies in particular $s \bar{s} s \bar{s}=s \bar{s}$ and $\bar{s} s \bar{s} s=\bar{s} s$ and thus $s \bar{s}$ and $\bar{s} s$ are idempotent. We also have

$$
(s \bar{s} s) \bar{s}(s \bar{s} s)=(s \bar{s})(s \bar{s})(s \bar{s}) s=s \bar{s} s \quad \text { and } \quad \bar{s}(s \bar{s} s) \bar{s}=\bar{s}
$$

Thus $\bar{s}$ is an inverse of $s \bar{s} s$.

Proposition 1.2 Let $s$ and $t$ be elements of a semigroup $S$ such that $s \mathcal{R}$ st (resp. ts $\mathcal{L} s)$. Then there exists a weak inverse $\bar{t}$ of $t$ such that $s t \bar{t}=s($ resp. $\bar{t} t s=s)$.

Proof. Since $s \mathcal{R}$ st, there exists an element $t^{\prime} \in S^{1}$ such that $s t t^{\prime}=s$. Let $\omega$ be an integer such that $\left(t t^{\prime}\right)^{\omega}$ is idempotent, and set $\bar{t}=t^{\prime}\left(t t^{\prime}\right)^{2 \omega-1}$. Then $s t \bar{t}=s t t^{\prime}\left(t t^{\prime}\right)^{2 \omega-1}=s$. Furthermore $\bar{t} t \bar{t}=t^{\prime}\left(t t^{\prime}\right)^{2 \omega-1} t t^{\prime}\left(t t^{\prime}\right)^{2 \omega-1}=t^{\prime}\left(t t^{\prime}\right)^{4 \omega-1}=$ $t^{\prime}\left(t t^{\prime}\right)^{2 \omega-1}=\bar{t}$. Thus $\bar{t}$ is a weak inverse of $t$. The proof for the $\mathcal{L}$ relation is dual.

A monoid $M$ divides a monoid $N$ if $M$ is a quotient of a submonoid of $N$. A variety of finite monoids is a class of finite monoids closed under taking submonoids, quotients and finite direct products.

Recall that a relational morphism between monoids $M$ and $N$ is a relation $\tau$ : $M \rightarrow N$ such that:

(1) $(m \tau)(n \tau) \subset(m n) \tau$ for all $m, n \in M$,

(2) $(m \tau)$ is non-empty for all $m \in M$,

(3) $1 \in 1 \tau$

Equivalently, $\tau$ is a relation whose graph

$$
\operatorname{graph}(\tau)=\{(m, n) \mid n \in m \tau\}
$$

is a submonoid of $M \times N$ that projects onto $M$.

Let $\mathbf{V}$ and $\mathbf{W}$ be varieties. The Malcev product of $\mathbf{V}$ and $\mathbf{W}$ is the variety $\mathbf{V}$ (ii) $\mathbf{W}$ defined as follows

$\mathbf{V}$ (M) $\mathbf{W}=\{M \mid$ There is a relational morphism $\tau: M \rightarrow N$ with $N \in \mathbf{W}$

$$
\text { and such that } \left.e \tau^{-1} \in \mathbf{V} \text { for all idempotents } e \in N\right\}
$$

Let $A$ be a finite set, called the alphabet, whose elements are letters. We denote by $A^{*}$ the free monoid over $A$. Elements of $A^{*}$ are words. In particular, the empty word, denoted by 1 , is the identity of $A^{*}$. A language is a subset of $A^{*}$. 
Let $M$ be a monoid and $L$ be a language of $A^{*}$. A monoid morphism $\varphi: A^{*} \rightarrow M$ recognizes a language $L$ if there exists a subset $P$ of $M$ such that $L=\varphi^{-1}(P)$. The syntactic congruence of $L$ is the equivalence $\sim_{L}$ on $A^{*}$ defined by

$$
u \sim_{L} v \text { if and only if, for every } x, y \in A^{*} \quad(x u y \in L \Longleftrightarrow x v y \in L) .
$$

The quotient $A^{*} / \sim_{L}$ is the syntactic monoid of $L$ and the natural morphism $\eta$ : $A^{*} \rightarrow M(L)$ is called the syntactic morphism: it recognizes $L$ and every surjective morphism $\varphi: A^{*} \rightarrow M$ that recognizes $L$ can be factorized through it, that is, there is a surjective morphism $\alpha: M \rightarrow M(L)$ such that $\eta=\alpha \circ \varphi$.

For technical reasons, it is more appropriate to use a variant of the concatenation product called the marked product. The results stated in the introduction refer to this product. Given a finite alphabet $A$ and a letter $a$ of $A$, the marked product of two subsets (also called languages) $L_{0}$ and $L_{1}$ of the free monoid $A^{*}$ is the language

$$
L_{0} a L_{1}=\left\{u \in A^{*} \mid u=u_{0} a u_{1} \text { for some } u_{0} \in L_{0} \text { and } u_{1} \in L_{1}\right\}
$$

Unambiguous, left and right deterministic products were introduced by Schützenberger. A product $L=L_{0} a L_{1}$ is unambiguous if every word $u$ of $L$ has a unique decomposition of the form $u=u_{0} a u_{1}$ with $u_{0} \in L_{0}$ and $u_{1} \in L_{1}$. It is left deterministic if every word of $L$ has exactly one prefix in $L_{0} a$. This means that in order to find the decomposition $u=u_{0} a u_{1}$ of a word of $L$, it suffices to read $u$ from left to right: the first prefix of $u$ in $L_{0} a$ will give $u_{0} a$, and thus the decomposition. Dually, a product $L=L_{0} a L_{1}$ is right deterministic if every word of $L$ has exactly one suffix in $a L_{1}$. A product is called bideterministic if it is both deterministic and antideterministic. Schützenberger [14] characterized the smallest variety of languages containing the language $\{1\}$ and closed under unambiguous (resp. deterministic, antideterministic) products. Later on, it was shown in $[8,9,12]$ that the closure of a variety of languages under unambiguous (resp. left deterministic, right deterministic) product correspond to the Malcev product $\mathbf{V} \rightarrow \mathbf{L I} \mathbb{M} \mathbf{V}$ (resp. $\mathbf{V} \rightarrow \mathbf{K}(\mathrm{M}) \mathbf{V}$, $\mathbf{V} \rightarrow \mathbf{K}^{\mathbf{r}}(\mathbb{M}) \mathbf{V}$ ), where $\mathbf{L I}, \mathbf{K}$ and $\mathbf{K}^{r}$ are respectively the varieties of semigroups $S$ such that, for every idempotent $e \in S, e S e=e,($ resp. $e S=e, S e=e)$.

\section{An expansion.}

In this section, we give the formal definition of our new expansion, which is related to certain special factorizations of words.

Let $M$ be a monoid, and let $\varphi: A^{*} \rightarrow M$ be a surjective (monoid) morphism. A good factorization (with respect to $\varphi$ ) is a triple $\left(x_{0}, a, x_{1}\right) \in A^{*} \times A \times A^{*}$ such that $\varphi\left(x_{0} a\right)<_{\mathcal{R}} \varphi\left(x_{0}\right)$ and $\varphi\left(a x_{1}\right)<_{\mathcal{L}} \varphi\left(x_{1}\right)$. A good factorization of a word $x \in A^{*}$ is a good factorization $\left(x_{0}, a, x_{1}\right)$ such that $x=x_{0} a x_{1}$. Two good factorizations $\left(x_{0}, a, x_{1}\right)$ and $\left(y_{0}, b, y_{1}\right)$ are equivalent if $\varphi\left(x_{0}\right)=\varphi\left(y_{0}\right), \varphi\left(x_{1}\right)=\varphi\left(y_{1}\right)$ and $a=b$. In particular, this implies $\varphi\left(x_{0} a x_{1}\right)=\varphi\left(y_{0} b y_{1}\right)$. Here is a first useful lemma.

Lemma 2.1 Let $\left(x_{0}, a, x_{1}\right)$ be a good factorization, let $x_{0}^{\prime}$ be a right factor of $x_{0}$ and let $x_{1}^{\prime}$ be a left factor of $x_{1}$. Then $\left(x_{0}^{\prime}, a, x_{1}^{\prime}\right)$ is a good factorization.

Proof. Set $x_{0}=x_{0}^{\prime \prime} x_{0}^{\prime}$ and $x_{1}=x_{1}^{\prime} x_{1}^{\prime \prime}$. If $\left(x_{0}^{\prime}, a, x_{1}^{\prime}\right)$ is not a good factorization, then $\varphi\left(x_{0}^{\prime} a\right) \mathcal{R} \varphi\left(x_{0}^{\prime}\right)$ or $\varphi\left(a x_{1}^{\prime}\right) \mathcal{L} \varphi\left(x_{1}^{\prime}\right)$. We treat the first case, but the other case is dual. Since $\mathcal{R}$ is stable on the left, we have $\varphi\left(x_{0}^{\prime \prime}\right) \varphi\left(x_{0}^{\prime} a\right) \mathcal{R} \varphi\left(x_{0}^{\prime \prime}\right) \varphi\left(x_{0}^{\prime}\right)$, whence $\varphi\left(x_{0}\right) \mathcal{R} \varphi\left(x_{0} a\right)$, a contradiction, since $\left(x_{0}, a, x_{1}\right)$ is a good factorization.

Define a relation $\sim_{\varphi}$ on $A^{*}$, by setting, for each $x, y \in A^{*}, x \sim_{\varphi} y$ if and only if the following three conditions are satisfied: 
(1) $\varphi(x)=\varphi(y)$,

(2) each good factorization of $x$ is equivalent to some good factorization of $y$,

(3) each good factorization of $y$ is equivalent to some good factorization of $x$.

Then one can state.

Proposition 2.2 The relation $\sim_{\varphi}$ is a congruence on $A^{*}$.

Proof. By symmetry, it suffices to prove that $x \sim_{\varphi} y$ implies $x b \sim_{\varphi} y b$ for each $b \in A$. Suppose that $x \sim_{\varphi} y$. Then $\varphi(x b)=\varphi(y b)$. By symmetry again, it suffices to show that each good factorization of $x b$ is equivalent to some good factorization of $y b$. Let $\left(x_{0}, a, x_{1}\right)$ be a good factorization of $x b$. If $x_{1}=1$, then $\left(x_{0}, a, x_{1}\right)=(x, b, 1)$, and $(y, a, 1)$ is a good factorization of $y b$ equivalent to $\left(x_{0}, a, x_{1}\right)$. Otherwise, $\left(x_{0}, a, x_{1}\right)=$ $\left(x_{0}, a, x_{1}^{\prime} b\right)$ for some $x_{1}^{\prime} \in A^{*}$, and by Lemma $2.1,\left(x_{0}, a, x_{1}^{\prime}\right)$ is a good factorization of $x$. Since $x \sim_{\varphi} y$, there exists an equivalent good factorization $\left(y_{0}, a, y_{1}^{\prime}\right)$ of $y$. We claim that $\left(y_{0}, a, y_{1}^{\prime} b\right)$ is a good factorization of $y b$ equivalent to $\left(x_{0}, a, x_{1}\right)$. The only thing to prove is that $\varphi\left(a y_{1}^{\prime} b\right)<_{\mathcal{L}} \varphi\left(y_{1}^{\prime} b\right)$. But this is clear, since

$$
\begin{aligned}
\varphi\left(a y_{1}^{\prime} b\right) & =\varphi(a) \varphi\left(y_{1}^{\prime}\right) \varphi(b)=\varphi(a) \varphi\left(x_{1}^{\prime}\right) \varphi(b) \\
& =\varphi(a) \varphi\left(x_{1}\right)<_{\mathcal{L}} \varphi\left(x_{1}\right)=\varphi\left(x_{1}^{\prime}\right) \varphi(b)=\varphi\left(y_{1}^{\prime} b\right) .
\end{aligned}
$$

Put $\widehat{M}=A^{*} / \sim_{\varphi}$ and let $\widehat{\varphi}: A^{*} \rightarrow \widehat{M}$ be the natural morphism induced by $\sim_{\varphi}$. By definition, $\widehat{\varphi}$ will be called the expansion of $\varphi$. Since $x \sim_{\varphi} y$ implies $\varphi(x)=\varphi(y)$, there exists a surjective morphism $\pi: \widehat{M} \rightarrow M$ such that the following diagram commutes:

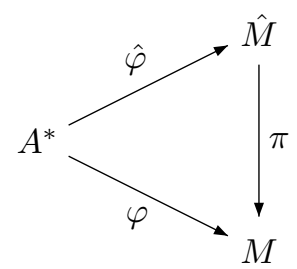

More generally, a monoid $\widehat{M}$ is said to be an expansion of a monoid $M$ if there exists a free monoid $A^{*}$ and a surjective morphism $\varphi: A^{*} \rightarrow M$ such that $\widehat{\varphi}: A^{*} \rightarrow \widehat{M}$ is the expansion of $\varphi$.

Example 2.1 Let $\varphi:\{a, b\}^{*} \rightarrow U_{1}=\{1,0\}$ be the surjective monoid morphism defined by $\varphi(a)=1$ and $\varphi(b)=0$. Then $(a a, b, 1)$ is a good factorization. The congruence $\sim_{\varphi}$ has three classes $C_{1}=\left\{\left.u|| u\right|_{b}=0\right\}, C_{2}=\left\{\left.u|| u\right|_{b}=1\right\}$, and $C_{3}=\left\{\left.u|| u\right|_{b}>1\right\}$. Therefore $\widehat{U}_{1}=\{1, s, 0\}$, where $s^{2}=0$, and $\widehat{\varphi}: A^{*} \rightarrow \widehat{U}_{1}$ is defined by $\widehat{\varphi}(a)=1$ and $\widehat{\varphi}(b)=s$. The morphism $\pi: \widehat{U}_{1} \rightarrow U_{1}$ is defined by $\pi(1)=1$ and $\pi(s)=\pi(0)=0$.

This example shows that our expansion is different from the expansion $\tilde{M}$ considered by Birget, Margolis and Rhodes in $[4,5]$. Indeed, one has $\tilde{U}_{1}=U_{1}$, but $\widehat{U}_{1} \neq U_{1}$.

Example 2.2 Let $G$ be a group and let $\varphi: A^{*} \rightarrow G$ be a surjective monoid morphism. Then there is no good factorization, and thus $\widehat{G}=G$.

We now verify the universal properties of our construction.

Proposition 2.3 Let $\varphi: A^{*} \rightarrow M, \varphi^{\prime}: A^{*} \rightarrow M^{\prime}$ and $\sigma: M^{\prime} \rightarrow M$ be surjective morphisms such that $\sigma \circ \varphi^{\prime}=\varphi$. Then there exists a surjective morphism $\widehat{\sigma}: \widehat{M}^{\prime} \rightarrow$ $\widehat{M}$ such that the following diagram commutes 


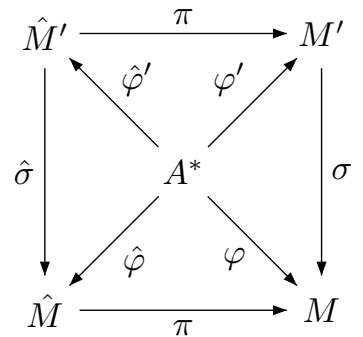

Proof. We have to show that $x \sim_{\varphi^{\prime}} y$ implies $x \sim_{\varphi} y$. First, $x \sim_{\varphi^{\prime}} y$ implies $\varphi^{\prime}(x)=\varphi^{\prime}(y)$, whence $\varphi(x)=\varphi(y)$. Let $\left(x_{0}, a, x_{1}\right)$ be a good factorization of $x$ with respect to $\varphi$. Then $\left(x_{0}, a, x_{1}\right)$ is also a good factorization of $x$ with respect to $\varphi^{\prime}$, because if $\varphi^{\prime}\left(x_{0} a\right) \mathcal{R} \varphi^{\prime}\left(x_{0}\right)$, then $\varphi\left(x_{0} a\right)=\sigma\left(\varphi^{\prime}\left(x_{0} a\right)\right) \mathcal{R} \sigma\left(\varphi^{\prime}\left(x_{0}\right)\right)=\varphi\left(x_{0}\right)$, and, dually, $\varphi^{\prime}\left(a x_{1}\right) \mathcal{L} \varphi^{\prime}\left(x_{1}\right)$ implies $\varphi\left(a x_{1}\right) \mathcal{L} \varphi\left(x_{1}\right)$. Since $x \sim_{\varphi^{\prime}} y$, there exists a good factorization of $y$ with respect to $\varphi^{\prime}$ equivalent to $\left(x_{0}, a, x_{1}\right)$, say $\left(y_{0}, a, y_{1}\right)$. Then $\varphi^{\prime}\left(x_{0}\right)=\varphi^{\prime}\left(y_{0}\right)$ whence $\varphi\left(x_{0}\right)=\varphi\left(y_{0}\right)$, and similarly, $\varphi\left(x_{1}\right)=\varphi\left(y_{1}\right)$. Therefore, $\left(y_{0}, a, y_{1}\right)$ is a good factorization of $y$ with respect to $\varphi$ equivalent to $\left(x_{0}, a, x_{1}\right)$. Dually, each good factorization of $y$ is equivalent to some good factorization of $x$, and $x \sim_{\varphi} y$. This proves the existence of a surjective morphism $\widehat{\sigma}: \widehat{M^{\prime}} \rightarrow \widehat{M}$ such that $\widehat{\sigma} \circ \widehat{\varphi^{\prime}}=\widehat{\varphi}$. It follows that

$$
\pi \circ \widehat{\sigma} \circ \widehat{\varphi}^{\prime}=\pi \circ \widehat{\varphi}=\varphi=\sigma \circ \varphi^{\prime}=\sigma \circ \pi^{\prime} \circ \widehat{\varphi}^{\prime}
$$

whence $\pi \circ \widehat{\sigma}=\sigma \circ \pi^{\prime}$ since $\widehat{\varphi^{\prime}}$ is surjective.

We now study the algebraic properties of our expansion. Recall that a semigroup is $n$-nilpotent if it satisfies the identity $s_{1} s_{2} \cdots s_{n}=t_{1} t_{2} \cdots t_{n}$, or equivalently, if $S$ has a zero, and if the set $S^{n}=\left\{s_{1} s_{2} \cdots s_{n} \mid s_{1}, \ldots, s_{n} \in S\right\}$ is equal to $\{0\}$.

Proposition 2.4 For every idempotent e of $M$, the semigroup $\pi^{-1}(e)$ is a 2-nilpotent semigroup.

Proof. Let $s, t \in \pi^{-1}(e)$ and let $x, y \in A^{*}$ be words such that $\widehat{\varphi}(x)=s$ and $\widehat{\varphi}(y)=t$. Then $\varphi(x)=\varphi(y)=e$. We claim that $x y$ has no good factorization. Indeed, let $\left(u_{0}, a, u_{1}\right)$ be a factorization of $x y$. By symmetry, we may assume that $x$ is a left factor of $u_{0}$. Set $u_{0}=x u_{0}^{\prime}$. Then

$$
\varphi(x)=\varphi(x) \varphi(x)=\varphi(x y)=\varphi\left(x u_{0}^{\prime} a u_{1}\right) \leq_{\mathcal{R}} \varphi\left(x u_{0}^{\prime} a\right) \leq_{\mathcal{R}} \varphi\left(x u_{0}^{\prime}\right) \leq_{\mathcal{R}} \varphi(x)
$$

whence $\varphi\left(x u_{0}^{\prime}\right) \mathcal{R} \varphi\left(x u_{0}^{\prime} a\right)$ and thus $\left(u_{0}, a, u_{1}\right)$ is not a good factorization of $x y$. Therefore the words of $\varphi^{-1}(e)$ which have no good factorization are $\sim_{\varphi}$-equivalent. It follows that $\pi^{-1}(e)$ satisfies the equation $s_{1} s_{2}=t_{1} t_{2}$ and thus is a 2-nilpotent semigroup.

Proposition 2.5 Let $s, s_{0}$ and $s_{1}$ be elements of $\widehat{M}$ such that $\pi\left(s_{0} s\right)=\pi\left(s_{0}\right)$ and $\pi\left(s s_{1}\right)=\pi\left(s_{1}\right)$. Then $s_{0} s s_{1}=s_{0} s_{1}$.

Proof. Let $x, x_{0}, x_{1} \in A^{*}$ be such that $\widehat{\varphi}(x)=s, \widehat{\varphi}\left(x_{0}\right)=s_{0}$ and $\widehat{\varphi}\left(x_{1}\right)=s_{1}$. By assumption, $\varphi\left(x_{0} x\right)=\varphi\left(x_{0}\right)$ and $\varphi\left(x x_{1}\right)=\varphi\left(x_{1}\right)$. We claim that $x_{0} x x_{1} \sim_{\varphi} x_{0} x_{1}$. Let $\left(u_{0}, a, u_{1}\right)$ be a good factorization of $x_{0} x x_{1}$. If $x=x_{0}^{\prime} a x_{1}^{\prime}$ for some words $x_{0}^{\prime}, x_{1}^{\prime}$ such that $u_{0}=x_{0} x_{0}^{\prime}$ and $u_{1}=x_{1}^{\prime} x_{1}$, then $\varphi\left(x_{0}\right)=\varphi\left(x_{0} x\right)<_{\mathcal{R}} \varphi\left(x_{0} x_{0}^{\prime}\right) \leq_{\mathcal{R}}$ $\varphi\left(x_{0}\right)$, a contradiction. Thus either $x_{0} x$ is a prefix or $u_{0}$ or $x x_{1}$ is a suffix of $u_{1}$. Suppose for instance $u_{0}=x_{0} x u_{0}^{\prime}$. Then since $\varphi\left(x_{0} x\right)=\varphi\left(x_{0}\right),\left(x_{0} u_{0}^{\prime}, a, u_{1}\right)$ is a good factorization of $x_{0} x_{1}$ equivalent with $\left(u_{0}, a, u_{1}\right)$. Conversely, let $\left(u_{0}, a, u_{1}\right)$ be a good factorization of $x_{0} x_{1}$. Then either $x_{0}$ is a prefix of $u_{0}$ or $x_{1}$ is a suffix of $u_{1}$. Suppose for instance $u_{0}=x_{0} u_{0}^{\prime}$. Then since $\varphi\left(x_{0} x\right)=\varphi\left(x_{0}\right),\left(x_{0} x u_{0}^{\prime}, a, u_{1}\right)$ is 
a good factorization of $x_{0} x x_{1}$ equivalent with $\left(u_{0}, a, u_{1}\right)$. This proves the claim and thus $s_{0} s s_{1}=\widehat{\varphi}\left(x_{0} x x_{1}\right)=\widehat{\varphi}\left(x_{0} x_{1}\right)=s_{0} s_{1}$.

We arrive at the main property of our expansion.

Theorem 2.6 The morphism $\pi: \widehat{M} \rightarrow M$ induces a bijection from $\mathrm{E}(\widehat{M})$ onto $\mathrm{E}(M)$, a bijection from $\operatorname{Reg}(\widehat{M})$ onto $\operatorname{Reg}(M)$ and an isomorphism from $\langle\operatorname{Reg}(\widehat{M})\rangle$ onto $\langle\operatorname{Reg}(M)\rangle$.

Proof. Since the image of a regular element is regular, $\pi(\langle\operatorname{Reg}(\widehat{M})\rangle)$ is certainly contained in $\langle\operatorname{Reg}(M)\rangle$. We first show that $\pi$ induces a surjection from $\operatorname{Reg}(\widehat{M})$ onto $\operatorname{Reg}(M)$. Let $r \in \operatorname{Reg}(M)$ and let $\bar{r}$ be an inverse of $r$. Let $s, t \in \widehat{M}$ be such that $\pi(s)=r$ and $\pi(t)=\bar{r}$. Then $\pi(s t)=r \bar{r}$ is idempotent, and by Proposition 2.4, $(s t)^{3}$ is idempotent. Therefore $x=(s t)^{3} s$ is a regular element such that $\pi(x)=r$. Similarly, $\pi$ induces a surjection from $\mathrm{E}(\widehat{M})$ onto $\mathrm{E}(M)$.

We now show that the restriction of $\pi$ to $\langle\operatorname{Reg}(\widehat{M})\rangle$ is injective. This will show that $\pi$ induces a bijection from $\mathrm{E}(\widehat{M})$ onto $\mathrm{E}(M)$, a bijection from $\operatorname{Reg}(\widehat{M})$ onto $\operatorname{Reg}(M)$ and an isomorphism from $\langle\operatorname{Reg}(\widehat{M})\rangle$ onto $\langle\operatorname{Reg}(M)\rangle$. Let $R$ be the set of all words which have no good factorizations. We claim that $R$ is a submonoid of $A^{*}$. Indeed, if $x, y \in R$, and if $\left(u_{0}, a, u_{1}\right)$ is a good factorization of $x y$, then either $x$ is a left factor of $u_{0}$, or $y$ is a right factor of $u_{1}$. Suppose, for instance, that $u_{0}=x u_{0}^{\prime}$ for some $u_{0}^{\prime} \in A^{*}$ (the other case is symmetrical). Then, by Lemma 2.1, $\left(u_{0}^{\prime}, a, u_{1}\right)$ is a good factorization of $y$, a contradiction. Thus $x y \in R$, proving the claim. Furthermore, it follows from the definition that $R$ is saturated by $\sim_{\varphi}$, and that two elements $r$ and $r^{\prime}$ of $R$ are congruent modulo $\sim_{\varphi}$ if and only if $\varphi(r)=\varphi\left(r^{\prime}\right)$. In particular, $\pi$ induces an isomorphism from $\widehat{\varphi}(R)$ onto $\varphi(R)$. Since $\widehat{\varphi}(R)$ is a submonoid of $\widehat{M}$, it remains to show that $\widehat{\varphi}(R)$ contains $\operatorname{Reg}(\widehat{M})$. This is the object of the next lemma.

Lemma 2.7 Let $x$ be a word such that $\widehat{\varphi}(x)$ is regular. Then $x$ has no good factorization.

Proof. . Let $s=\widehat{\varphi}(x)$. Since $s$ is regular, there exists an element $\bar{s} \in M$ such that $s \bar{s} s=s$. Let $\bar{x}$ be a word of $A^{*}$ such that $\widehat{\varphi}(\bar{x})=\bar{s}$. In particular, $\widehat{\varphi}(x \bar{x} x)=\widehat{\varphi}(x)$. Suppose that $x$ has a good factorization. Then since $x \bar{x} x \sim_{\varphi} x, x \bar{x} x$ also has a good factorization. There are three possible types for this factorization, depending on the place of the middle letter

(1) $\left(x_{0}, a, x_{1} \bar{x} x\right)$, where $x_{0} a x_{1}=x$,

(2) $\left(x \bar{x}_{0}, a, \bar{x}_{1} x\right)$, where $\bar{x}_{0} a \bar{x}_{1}=\bar{x}$,

(3) $\left(x \bar{x} x_{0}, a, x_{1}\right)$, where $x_{0} a x_{1}=x$.

But (1) is not a good factorization, since $\varphi\left(x_{1} \bar{x} x\right) \mathcal{L} \varphi\left(a x_{1} \bar{x} x\right),(2)$ is not a good factorization, since $\varphi\left(x \bar{x}_{0}\right) \mathcal{R} \varphi\left(x \bar{x}_{0} a\right)$, and finally (3) is not a good factorization, since $\varphi\left(x \bar{x} x_{0}\right) \mathcal{R} \varphi\left(x \bar{x} x_{0} a\right)$. Therefore $x$ cannot have any good factorization. This concludes the proofs of Lemma 2.7 and Theorem 2.6.

\section{Monoids in which the regular elements form a submonoid}

The results of the previous section can be made precise if the regular elements of $M$ form a submonoid of $M$. This condition is satisfied in particular if the product of two idempotents is an idempotent. 
Proposition 3.1 Let $S$ be a semigroup in which the idempotents form a subsemigroup. Then the regular elements of $S$ form a subsemigroup of $S$.

Proof. Let $r_{1}$ and $r_{2}$ be regular elements of $S$. Then there exist idempotents $e$ and $f$ such that $e \mathcal{L} r_{1}$ and $r_{2} \mathcal{R} f$. Therefore, there exist $x, y \in S^{1}$ such that

$$
x r_{1}=e \quad r_{1} e=r_{1} \quad r_{2} y=f \quad f r_{2}=r_{2}
$$

whence $r_{1} r_{2}=r_{1}$ ef $r_{2}$. It follows that ef $\mathcal{J} r_{1} r_{2}$ since

$$
x\left(r_{1} e f r_{2}\right) y=\left(x r_{1}\right)(e f)\left(r_{2} y\right)=e(e f) f=e f .
$$

Thus $r_{1} r_{2}$ is regular.

We need the following property of the semigroups in which the regular elements form a subsemigroup.

Lemma 3.2 Let $S$ be a semigroup in which the regular elements form a subsemigroup. If $\bar{s}_{1}$ (resp. $\left.\bar{s}_{2}\right)$ is a weak inverse of $s_{1}$ (resp. $\left.s_{2}\right)$, then there exist a weak inverse $\bar{s}$ of $s_{1} s_{2}$ such that $\left(s_{1} \bar{s}_{1} s_{1}\right)\left(s_{2} \bar{s}_{2} s_{2}\right)=\left(s_{1} \bar{s}_{1}\right)\left(s_{1} s_{2}\right) \bar{s}\left(s_{1} s_{2}\right)\left(\bar{s}_{2} s_{2}\right)$.

Proof. By Proposition 1.1, $s_{1} \bar{s}_{1} s_{1}$ and $s_{2} \bar{s}_{2} s_{2}$ are regular, and thus

$$
s=\left(s_{1} \bar{s}_{1} s_{1}\right)\left(s_{2} \bar{s}_{2} s_{2}\right)
$$

is also regular and has an inverse $s^{\prime}$. Set $\bar{s}=\bar{s}_{2} s_{2} s^{\prime} s_{1} \bar{s}_{1}$. We get $s_{1} \bar{s}_{1} s_{1} s_{2} \bar{s} s_{1} s_{2} \bar{s}_{2} s_{2}=$ $s s^{\prime} s=s$. Furthermore $\bar{s}$ is a weak inverse of $s_{1} s_{2}$ since

$$
\bar{s} s_{1} s_{2} \bar{s}=\bar{s}_{2} s_{2} s^{\prime} s_{1} \bar{s}_{1} s_{1} s_{2} \bar{s}_{2} s_{2} s^{\prime} s_{1} \bar{s}_{1}=\bar{s}_{2} s_{2} s^{\prime} s s^{\prime} s_{1} \bar{s}_{1}=\bar{s}_{2} s_{2} s^{\prime} s_{1} \bar{s}_{1}=\bar{s} .
$$

Theorem 3.3 Let $M$ be a monoid in which the regular elements form a submonoid. Let $s_{1}, \ldots, s_{n}$ be elements of $M$ such that, for $1 \leq i \leq n$, either $s_{1} \cdots s_{i-1} \mathcal{R} s_{1} \cdots s_{i}$ or $s_{i} \cdots s_{n} \mathcal{L} s_{i+1} \cdots s_{n}$. Then $s_{1} s_{2} \cdots s_{n}$ is regular.

Proof. We prove the result by induction on $n$. If $n=1$, we have either $1 \mathcal{R} s_{1}$ or $s_{1} \mathcal{L} 1$ and thus $s_{1}$ is regular. Assume that for some $i<n$ we have $s_{1} \cdots s_{i-1} \mathcal{R}$ $s_{1} \cdots s_{i}$ and $s_{1} \cdots s_{i} \mathcal{R} s_{1} \cdots s_{i+1}$. Then $s_{1} \cdots s_{i-1} \mathcal{R} s_{1} \cdots s_{i-1}\left(s_{i} s_{i+1}\right)$ and one may apply the induction hypothesis to the sequence $s_{1}, \ldots, s_{i-1},\left(s_{i} s_{i+1}\right), s_{i+2}, \ldots, s_{n}$ to show that $s_{1} s_{2} \cdots s_{n}$ is regular.

Next assume that $1 \mathcal{R} s_{1}$. Then $s_{1}$ is a unit in $M$ and thus every relation of the form $s_{1} \cdots s_{i-1} \mathcal{R} s_{1} \cdots s_{i}$ is equivalent with $s_{2} \cdots s_{i-1} \mathcal{R} s_{2} \cdots s_{i}$. Therefore, induction applied to the sequence $s_{2}, \ldots, s_{n}$ shows that $s_{2} \cdots s_{n}$ is regular. Since $s_{1}$ is regular, $s_{1} s_{2} \cdots s_{n}$ is also regular.

A dual argument can be applied to the $\mathcal{L}$ relation, and thus we are left with the case where the following relations hold:

$$
\begin{array}{ccc}
s_{1} s_{2} \cdots s_{n} & \mathcal{L} s_{2} \cdots s_{n} & (i=1) \\
& s_{1} \mathcal{R} s_{1} s_{2} & \\
s_{3} s_{4} \cdots s_{n} & \mathcal{L} s_{4} \cdots s_{n} & (i=2) \\
s_{1} s_{2} s_{3} & \mathcal{R} s_{1} s_{2} s_{3} s_{4} & (i=3) \\
\vdots & & (i=4) \\
s_{1} \cdots s_{n-1} & \mathcal{R} s_{1} s_{2} \cdots s_{n} & (i=n)
\end{array}
$$

This implies in particular that $n$ is even. By Proposition 1.2, there exist for $1 \leq i \leq n$ a weak inverse $\bar{s}_{i}$ of $s_{i}$ such that

$$
\begin{aligned}
& s_{i+1} \cdots s_{n}=\bar{s}_{i} s_{i}\left(s_{i+1} \cdots s_{n}\right) \quad \text { if } i \text { is odd } \\
& \left.s_{1} \cdots s_{i-1}=\left(\begin{array}{lll}
s_{1} & \cdots & s_{i-1}
\end{array}\right) s_{i} \bar{s}_{i} \quad \text { if } i \text { is even }\right\}
\end{aligned}
$$


Set, for $1 \leq j \leq n, s_{1, j}=s_{1} \cdots s_{j}$. We show by induction on $j$ that for $1 \leq j \leq n$, there exist weak inverses $\bar{s}_{1, j}$ of $s_{1, j}$ such that

$$
s_{1} \cdots s_{n}=s_{1,1} \bar{s}_{1,1} s_{1,2} \bar{s}_{1,2} s_{1,3} \bar{s}_{1,3} \cdots s_{1, j} \bar{s}_{1, j} s_{1, j} s_{j+1} \cdots s_{n}
$$

First apply (3.1) with $i=1$. We get $s_{2} \cdots s_{n}=\bar{s}_{1} s_{1} s_{2} \cdots s_{n}$ and thus $s_{1} s_{2} \cdots s_{n}=$ $s_{1} \bar{s}_{1} s_{1} s_{2} \cdots s_{n}$, proving (3.2) for $j=1$. By induction, assume that the result holds for $j$. If $j+1$ is odd, we have by (3.1)

$$
\left(s_{j+1} \bar{s}_{j+1} s_{j+1}\right)\left(s_{j+2} \cdots s_{n}\right)=s_{j+1}\left(s_{j+2} \cdots s_{n}\right)
$$

Therefore

$$
s_{1, j} \bar{s}_{1, j} s_{1, j} s_{j+1} \cdots s_{n}=\left(s_{1, j} \bar{s}_{1, j} s_{1, j}\right)\left(s_{j+1} \bar{s}_{j+1} s_{j+1}\right)\left(s_{j+2} \cdots s_{n}\right)
$$

Now by Lemma 3.2, there exists a weak inverse $\bar{s}_{1, j+1}$ of $s_{1, j} s_{j+1}=s_{1, j+1}$ such that

$$
\begin{aligned}
\left(s_{1, j} \bar{s}_{1, j} s_{1, j}\right)\left(s_{j+1} \bar{s}_{j+1} s_{j+1}\right) & =\left(s_{1, j} \bar{s}_{1, j}\right)\left(s_{1, j} s_{j+1}\right) \bar{s}_{1, j+1}\left(s_{1, j} s_{j+1}\right)\left(\bar{s}_{j+1} s_{j+1}\right) \\
& =\left(s_{1, j} \bar{s}_{1, j}\right)\left(s_{1, j+1} \bar{s}_{1, j+1} s_{1, j+1}\right)\left(\bar{s}_{j+1} s_{j+1}\right)
\end{aligned}
$$

It follows that

$$
\begin{aligned}
s_{1, j} \bar{s}_{1, j} s_{1, j} s_{j+1} \cdots s_{n} & =\left(s_{1, j} \bar{s}_{1, j}\right)\left(s_{1, j+1} \bar{s}_{1, j+1} s_{1, j+1}\right)\left(\bar{s}_{j+1} s_{j+1}\right)\left(s_{j+2} \cdots s_{n}\right) \\
& =\left(s_{1, j} \bar{s}_{1, j}\right)\left(s_{1, j+1} \bar{s}_{1, j+1}\right)\left(s_{1, j+1} s_{j+2} \cdots s_{n}\right) \quad \text { by }(3.3)
\end{aligned}
$$

and thus (3.2) holds for $j+1$.

If $j+1$ is even, we have by (3.1)

$$
s_{1, j} s_{j+1}=s_{1} \cdots s_{j} s_{j+1}=s_{1} \cdots s_{j} s_{j+1} \bar{s}_{j+1} s_{j+1}=s_{1, j} s_{j+1} \bar{s}_{j+1} s_{j+1}
$$

Therefore by the induction hypothesis,

$$
\begin{aligned}
& s_{1} \cdots s_{n}=s_{1,1} \bar{s}_{1,1} s_{1,2} \bar{s}_{1,2} \cdots s_{1, j} \bar{s}_{1, j} s_{1, j} s_{j+1} \cdots s_{n} \\
& =s_{1,1} \bar{s}_{1,1} s_{1,2} \bar{s}_{1,2} \cdots s_{1, j} \bar{s}_{1, j} s_{1, j} s_{j+1} \bar{s}_{j+1} s_{j+1} \cdots s_{n}
\end{aligned}
$$

Now by Lemma 3.2 , there exists a weak inverse $\bar{s}_{1, j+1}$ of $s_{1, j} s_{j+1}=s_{1, j+1}$ such that

$$
\begin{aligned}
\left(s_{1, j} \bar{s}_{1, j} s_{1, j}\right)\left(s_{j+1} \bar{s}_{j+1} s_{j+1}\right) & =\left(s_{1, j} \bar{s}_{1, j}\right)\left(s_{1, j} s_{j+1}\right) \bar{s}_{1, j+1}\left(s_{1, j} s_{j+1}\right)\left(\bar{s}_{j+1} s_{j+1}\right) \\
& =\left(s_{1, j} \bar{s}_{1, j}\right)\left(s_{1, j+1} \bar{s}_{1, j+1} s_{1, j+1}\right)\left(\bar{s}_{j+1} s_{j+1}\right)
\end{aligned}
$$

It follows that

$$
\begin{aligned}
& s_{1} \cdots s_{n}=s_{1,1} \bar{s}_{1,1} s_{1,2} \bar{s}_{1,2} \cdots\left(s_{1, j} \bar{s}_{1, j}\right)\left(s_{1, j+1} \bar{s}_{1, j+1} s_{1, j+1}\right)\left(\bar{s}_{j+1} s_{j+1}\right) s_{j+2} \cdots s_{n} \\
& =s_{1,1} \bar{s}_{1,1} s_{1,2} \bar{s}_{1,2} \cdots\left(s_{1, j} \bar{s}_{1, j}\right)\left(s_{1, j+1} \bar{s}_{1, j+1} s_{1, j+1}\right) s_{j+2} \cdots s_{n} \quad \text { by }(3.4)
\end{aligned}
$$

and thus (3.2) also holds for $j+1$. In particular, (3.2) gives for $j=n$,

$$
s_{1} \cdots s_{n}=\left(s_{1,1} \bar{s}_{1,1}\right)\left(s_{1,2} \bar{s}_{1,2}\right) \cdots\left(s_{1, n} \bar{s}_{1, n}\right) s_{1, n}
$$

and thus $s_{1} \cdots s_{n}$ is regular, as a product of the regular elements $s_{1,1} \bar{s}_{1,1}, \ldots$, $\bar{s}_{1, n-1} s_{1, n-1}$ and $s_{1, n} \bar{s}_{1, n} s_{1, n}$.

If $M$ is a monoid in which the regular elements form a submonoid, Lemma 2.7 can be completed as follows.

Proposition 3.4 Let $\varphi: A^{*} \rightarrow M$ be a surjective monoid morphism, where $M$ is a monoid in which the regular elements form a submonoid. A word $x \in A^{*}$ has a good factorization if and only if $\widehat{\varphi}(x)$ is non-regular. 
Proof. If $x$ has a good factorization, then $\widehat{\varphi}(x)$ is non-regular by Lemma 2.7. Conversely, suppose that $\widehat{\varphi}(x)$ is non-regular. If $\varphi(x)$ is regular, then there exists by Theorem 2.6 a word $y$ such that $\widehat{\varphi}(y)$ is regular and $\varphi(x)=\varphi(y)$. On the other hand, $\widehat{\varphi}(x) \neq \widehat{\varphi}(y)$ since $\widehat{\varphi}(x)$ is non-regular. Therefore, since $y$ has no good factorization by Lemma 2.7, $x$ must have a good factorization, otherwise $x \sim_{\varphi} y$ and $\widehat{\varphi}(x)=\widehat{\varphi}(y)$.

Now suppose $\varphi(x)$ non-regular. Let $x=a_{1} \cdots a_{k}$, with $a_{1}, \ldots, a_{k} \in A$, and set for $1 \leq i \leq k, s_{i}=\varphi\left(a_{i}\right)$. If $x$ has no good factorization, then in particular $\left(a_{1} \cdots a_{i-1}, a_{i}, a_{i+1} \cdots a_{k}\right)$ is not a good factorization of $x$, whence

either $\varphi\left(a_{1}\right) \cdots \varphi\left(a_{i-1}\right) \mathcal{R} \varphi\left(a_{1}\right) \cdots \varphi\left(a_{i}\right)$ or $\varphi\left(a_{i+1}\right) \cdots \varphi\left(a_{k}\right) \mathcal{L} \varphi\left(a_{i}\right) \cdots \varphi\left(a_{k}\right)$.

Now by Theorem $3.3, \varphi(x)=\varphi\left(a_{1} \cdots a_{k}\right)$ is regular, a contradiction.

This last result has an important consequence. Let $M$ be a monoid in which the regular elements form a submonoid and let $\alpha: A^{*} \rightarrow M$ be a surjective morphism. Suppose that $M$ has a zero and a 0 -minimal null ideal $J$ and let $\beta: M \rightarrow M / J$ be the natural morphism. Finally, set $\varphi=\beta \circ \alpha: A^{*} \rightarrow M / J$ and let $\widehat{M / J}$ be the expansion of $M / J$ relative to $\varphi$. These notations are summarized in the following diagram.

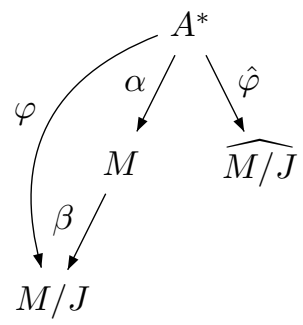

Theorem 3.5 With the previous notations, $M$ is a quotient of $\widehat{M / J}$.

Proof. Set $K=J \backslash\{0\}$. It suffices to show that, for every $u, v \in A^{*}, u \sim_{\varphi} v$ implies $\alpha(u)=\alpha(v)$. Since $u \sim_{\varphi} v$ implies $\varphi(u)=\varphi(v)$, it suffices to consider the case $\alpha(u), \alpha(v) \in J$.

Lemma 3.6 Let $x$ be a word such that $\alpha(x) \in K$. Then $x$ has a good factorization.

Proof. Let $x=a_{1} \cdots a_{k}$, with $a_{1}, \ldots, a_{k} \in A$, and put $s_{i}=\alpha\left(a_{i}\right)$ for $1 \leq i \leq k$. First assume that $s_{i} \in K$ for some $i$. We claim that $f_{i}=\left(a_{1} \cdots a_{i-1}, a_{i}, a_{i+1} \cdots a_{k}\right)$ is a good factorization of $x$. Indeed, $\alpha\left(a_{1} \cdots a_{i-1}\right) \notin K$, otherwise

$$
\alpha(x)=\alpha\left(a_{1} \cdots a_{i-1}\right) \alpha\left(a_{i}\right) \alpha\left(a_{i+1} \cdots a_{k}\right)=0
$$

since $J$ is null. Similarly, $\alpha\left(a_{i+1} \cdots a_{k}\right) \notin K$, and thus $\varphi\left(a_{1} \cdots a_{i-1}\right) \neq 0, \varphi\left(a_{i}\right)=0$, $\varphi\left(a_{i+1} \cdots a_{k}\right) \neq 0$, proving the claim.

Suppose now $s_{i} \notin K$ for $1 \leq i \leq k$. Then $\varphi\left(a_{i}\right)=\alpha\left(a_{i}\right)=s_{i}$. If $x$ has no good factorization, then none of the factorizations $f_{i}$ is good. It follows that for $1 \leq i \leq k$, either $s_{1} \cdots s_{i-1} \mathcal{R} s_{1} \cdots s_{i}$ or $s_{i+1} \cdots s_{k} \mathcal{L} s_{i} \cdots s_{k}$. Therefore by Theorem 3.3, $\alpha(x)=s_{1} \cdots s_{k}$ is regular, a contradiction.

We now complete the proof of Theorem 3.5. If $\alpha(u) \in K$, (or if $\alpha(v) \in K$ ), $u$ (or $v$ ) has a good factorization by Lemma 3.6. Since $u \sim_{\varphi} v, u$ and $v$ have equivalent factorizations, $\left(u_{0}, a, u_{1}\right)$ and $\left(v_{0}, a, v_{1}\right)$ respectively. Since $\varphi(u)<_{\mathcal{R}} \varphi\left(u_{0}\right), \varphi\left(u_{0}\right) \neq$ 0 , whence $\varphi\left(u_{0}\right)=\alpha\left(u_{0}\right)$. Similarly $\varphi\left(u_{1}\right)=\alpha\left(u_{1}\right), \varphi\left(v_{0}\right)=\alpha\left(v_{0}\right)$ and $\varphi\left(v_{1}\right)=$ 
$\alpha\left(v_{1}\right)$. Now since $\left(u_{0}, a, u_{1}\right)$ and $\left(v_{0}, a, v_{1}\right)$ are equivalent factorizations, $\varphi\left(u_{0}\right)=$ $\varphi\left(v_{0}\right)$ and $\varphi\left(u_{1}\right)=\varphi\left(v_{1}\right)$. It follows that

$$
\begin{aligned}
& \alpha(u)=\alpha\left(u_{0} a u_{1}\right)=\alpha\left(u_{0}\right) \alpha(a) \alpha\left(u_{1}\right)=\varphi\left(u_{0}\right) \alpha(a) \varphi\left(u_{1}\right) \\
& =\varphi\left(v_{0}\right) \alpha(a) \varphi\left(v_{1}\right)=\alpha\left(v_{0}\right) \alpha(a) \alpha\left(v_{1}\right)=\alpha\left(v_{0} a v_{1}\right)=\alpha(v) .
\end{aligned}
$$

Finally if $\alpha(u) \notin K$ and $\alpha(v) \notin K$, then $\alpha(u)=\alpha(v)=0$.

\section{Bideterministic concatenation product}

We now apply the results of the previous sections to the study of the bideterministic concatenation product.

Theorem 4.1 Let $\varphi: A^{*} \rightarrow M$ be a surjective morphism. Every language recognized by $\hat{\varphi}$ is a finite boolean combination of languages recognized by $M$ or of bideterministic products of the form $L_{0} a L_{1}$, where $L_{0}$ and $L_{1}$ are recognized by $M$.

Proof. Since any language recognized by $\widehat{\varphi}$ is a finite union of sets of the form $\widehat{\varphi}^{-1}(s)$, it suffices to establish the result for $L=\widehat{\varphi}^{-1}(s)$, where $s \in \widehat{M}$. Set $m=\pi(s)$, and fix an element $x$ of $L$. Put

$$
\begin{aligned}
T=\left\{\left(m_{0}, a, m_{1}\right) \in M \times A \times M \mid m_{0} \varphi(a) m_{1}=m, m_{0} \varphi(a)<\mathcal{R} m_{0}\right. \text { and } & \\
& \left.\varphi(a) m_{1}<_{\mathcal{L}} m_{1}\right\} .
\end{aligned}
$$

This set is the disjoint union of two sets $E$ and $F$, defined as follows

$$
\begin{array}{r}
E=\left\{\left(m_{0}, a, m_{1}\right) \in T \mid \text { there exists a good factorization }\left(x_{0}, a, x_{1}\right) \text { of } x\right. \\
\text { such that } \left.\varphi\left(x_{0}\right)=m_{0} \text { and } \varphi\left(x_{1}\right)=m_{1}\right\}, \\
F=\left\{\left(m_{0}, a, m_{1}\right) \in T \mid \text { there exists no good factorization }\left(x_{0}, a, x_{1}\right) \text { of } x\right. \\
\text { such that } \left.\varphi\left(x_{0}\right)=m_{0} \text { and } \varphi\left(x_{1}\right)=m_{1}\right\} .
\end{array}
$$

Note that the definition of $E$ and $F$ depends on $s$, but does not depend on the choice of $x$ in $L$, since all the elements of $L$ are congruent modulo $\sim_{\varphi}$. We claim that

$$
L=\varphi^{-1}(m) \cap\left(\left[\bigcap_{\left(m_{0}, a, m_{1}\right) \in E} \varphi^{-1}\left(m_{0}\right) a \varphi^{-1}\left(m_{1}\right)\right] \backslash\left[\bigcup_{\left(m_{0}, a, m_{1}\right) \in F} \varphi^{-1}\left(m_{0}\right) a \varphi^{-1}\left(m_{1}\right)\right]\right) .
$$

First $\varphi^{-1}(m)=\widehat{\varphi}^{-1}\left(\pi^{-1}(m)\right)$ contains $\widehat{\varphi}^{-1}(s)=L$. Let $u \in \varphi^{-1}(m)$ and let $\left(m_{0}, a, m_{1}\right) \in T$. Then $u \in \varphi^{-1}\left(m_{0}\right) a \varphi^{-1}\left(m_{1}\right)$ if and only if $u$ has a good factorization $\left(u_{0}, a, u_{1}\right)$ such that $\varphi\left(u_{0}\right)=m_{0}$ and $\varphi\left(u_{1}\right)=m_{1}$. Therefore,

$$
u \in \bigcap_{\left(m_{0}, a, m_{1}\right) \in E} \varphi^{-1}\left(m_{0}\right) a \varphi^{-1}\left(m_{1}\right)
$$

if and only if each good factorization of $x$ is equivalent to a good factorization of $u$, and

$$
u \in A^{*} \backslash \bigcup_{\left(m_{0}, a, m_{1}\right) \in F} \varphi^{-1}\left(m_{0}\right) a \varphi^{-1}\left(m_{1}\right)
$$

if and only if each good factorization of $u$ is equivalent to a good factorization of $x$. Therefore $u$ satisfies (1), (2) and $\varphi(u)=m$, if and only if $u \sim_{\varphi} x$, or equivalently, if $u \in L$. This proves the claim and the theorem.

Let $\varphi_{0}: A^{*} \rightarrow M_{0}$ and $\varphi_{1}: A^{*} \rightarrow M_{1}$ be two surjective morphisms recognizing the languages $L_{0}$ and $L_{1}$, respectively, and let $a$ be a letter of $A$. Let $\varphi: A^{*} \rightarrow$ $M_{0} \times M_{1}$ be the morphism defined by $\varphi(u)=\left(\varphi_{0}(u), \varphi_{1}(u)\right)$, and let $M=\varphi\left(A^{*}\right)$. Then we can state 
Theorem 4.2 If the product $L=L_{0} a L_{1}$ is bideterministic, then $L$ is recognized by $\widehat{\varphi}$.

Proof. Put $L=L_{0} a L_{1}$. It suffices to show that $x \sim_{\varphi} y$ implies $x \sim_{L} y$. First, $x \sim_{\varphi} y$ implies $\varphi_{0}(x)=\varphi_{0}(y)$ and $\varphi_{1}(x)=\varphi_{1}(y)$. Suppose that $u x v \in L$. Three cases may occur

(1) $u x v=u_{0} a u_{1} x v$, with $u_{0} a u_{1}=u, u_{0} \in L_{0}$ and $u_{1} x v \in L_{1}$. Then $x \sim_{L_{1}} y$ since $\varphi_{1}(x)=\varphi_{1}(y)$, and thus $u_{1} y v \in L_{1}$ and $u y v=u_{0} a u_{1} y v \in L$.

(2) $u x v=u x v_{0} a v_{1}$, with $v_{0} a v_{1}=v, u x v_{0} \in L_{0}$ and $v_{1} \in L_{1}$. This case is symmetrical to (1) and thus $u y v \in L$.

(3) $u x v=u x_{0} a x_{1} v$, with $x_{0} a x_{1}=x, u x_{0} \in L_{0}$ and $x_{1} v \in L_{1}$. If $\varphi_{0}\left(u x_{0} a\right) \mathcal{R}$ $\varphi_{0}\left(u x_{0}\right)$, there exists a word $t$ such that $\varphi_{0}\left(u x_{0} a t\right)=\varphi_{0}\left(u x_{0}\right)$, whence $u x_{0} a t \in$ $L_{0}$ and $L_{0} a A^{*} \cap L_{0} \neq \emptyset$. Therefore, $\varphi_{0}\left(u x_{0} a\right)<_{\mathcal{R}} \varphi_{0}\left(u x_{0}\right)$, whence $\varphi\left(u x_{0} a\right)<_{\mathcal{R}}$ $\varphi\left(u x_{0}\right)$ and dually $\varphi\left(a x_{1} v\right)<_{\mathcal{L}} \varphi\left(x_{1} v\right)$. It follows that $\left(u x_{0}, a, x_{1} v\right)$ is a good factorization of $u x v$. Now $x \sim_{\varphi} y$ implies $u x v \sim_{\varphi} u y v$, and there exists a good factorization $\left(w_{0}, a, w_{1}\right)$ of $u y v$ equivalent to $\left(u x_{0}, a, x_{1} v\right)$. In particular, $\varphi\left(w_{0}\right)=\varphi\left(u x_{0}\right)$, whence $\varphi_{0}\left(w_{0}\right)=\varphi_{0}\left(u x_{0}\right)$, and $w_{0} \in L_{0}$. Similarly, $w_{1} \in L_{1}$ and $u y v=w_{0} a w_{1} \in L$. Thus $u x v \in L$ implies $u y v \in L$. Now, $u y v \in L$ implies $u x v \in L$ by a dual argument and $x \sim_{L} y$.

The previous results can be converted into results on varieties. Let $\mathbf{V}$ be a variety of monoids and let $\mathcal{V}$ be the corresponding variety of languages. We denote by $\widehat{\mathbf{V}}$ the variety of monoids generated by the monoids $\widehat{M}$ for $M \in \mathbf{V}$. Finally, we denote by $\widehat{\mathcal{V}}$ the variety of languages associated with $\widehat{\mathbf{V}}$.

Theorem 4.3 For each alphabet $A, \widehat{\mathcal{V}}\left(A^{*}\right)$ is the boolean algebra generated by languages of the form $L$ or $L_{0} a L_{1}$, where $L, L_{0}$ and $L_{1}$ are in $\mathcal{V}\left(A^{*}\right)$ and $L_{0} a L_{1}$ is a bideterministic product.

Proof. Let $\mathcal{C}\left(A^{*}\right)$ be the boolean algebra generated by languages of the form $L$ or $L_{0} a L_{1}$, where $L, L_{0}$ and $L_{1}$ are in $\mathcal{V}\left(A^{*}\right)$ and $L_{0} a L_{1}$ is a bideterministic product. We claim that $\mathcal{C}\left(A^{*}\right)$ is contained in $\widehat{\mathcal{V}}\left(A^{*}\right)$. By definition of a variety of languages, $\widehat{\mathcal{V}}\left(A^{*}\right)$ is a boolean algebra of languages and since $\mathbf{V}$ is a subvariety of $\widehat{\mathbf{V}}, \widehat{\mathcal{V}}\left(A^{*}\right)$ contains the languages of $\mathcal{V}\left(A^{*}\right)$. Let $L_{0} a L_{1}$ be a bideterministic product with $L_{0}$ and $L_{1}$ in $\mathcal{V}\left(A^{*}\right)$. Let $\varphi_{0}: A^{*} \rightarrow M_{0}$ and $\varphi_{1}: A^{*} \rightarrow M_{1}$ be two surjective morphisms recognizing the languages $L_{0}$ and $L_{1}$, respectively, with $M_{0}, M_{1} \in \mathbf{V}$. By Theorem 4.2, $L_{0} a L_{1}$ is recognized by a monoid $\widehat{M}$, where $M$ is a submonoid of $M_{0} \times M_{1}$. Since $\mathbf{V}$ is a variety, it follows that $M \in \mathbf{V}$ and $\widehat{M} \in \widehat{\mathbf{V}}$, and thus $L_{0} a L_{1} \in \widehat{\mathcal{V}}\left(A^{*}\right)$, proving the claim.

We now show that $\widehat{\mathcal{V}}\left(A^{*}\right)$ is contained in $\mathcal{C}\left(A^{*}\right)$. Let $L \in \widehat{\mathcal{V}}\left(A^{*}\right)$. By definition, $L$ is recognized by a monoid $\widehat{M}$, where $M \in \mathbf{V}$. Therefore, there exist a morphism $\alpha: A^{*} \rightarrow \widehat{M}$ such that $L=\alpha^{-1}(P)$, where $P=\alpha(L)$, and a surjective morphism $\varphi$ : $B^{*} \rightarrow M$ which defines the expansion $\widehat{\varphi}: B^{*} \rightarrow \widehat{M}$. Then, by the universal property of the free monoid $A^{*}$, there exists a morphism $\beta: A^{*} \rightarrow B^{*}$ such that $\alpha=\widehat{\varphi} \circ \beta$. In particular $L=\beta^{-1}\left(\widehat{\varphi}^{-1}(P)\right)$. Now, $\widehat{\varphi}^{-1}(P)$ is recognized by $\widehat{\varphi}$ and thus, by Theorem 4.1 , is in $\mathcal{C}\left(B^{*}\right)$. Therefore, it suffices to show that $\beta^{-1}\left(\mathcal{C}\left(B^{*}\right)\right) \subset \mathcal{C}\left(A^{*}\right)$. Since $\beta^{-1}$ commutes with boolean operations, we need only consider the generators of $\mathcal{C}\left(B^{*}\right)$. If $K$ is a language of $\mathcal{V}\left(B^{*}\right)$, then $\beta^{-1}(K) \in \mathcal{V}\left(A^{*}\right)$ by definition of a variety of languages. Therefore, it only remains to verify that if $K_{0} b K_{1}$ is a bideterministic product, where $b \in B$ and $K_{0}, K_{1} \in \mathcal{V}\left(B^{*}\right)$, then $\beta^{-1}\left(K_{0} b K_{1}\right) \in \mathcal{C}\left(A^{*}\right)$. Given a subset $S$ of $B^{*}$ and a word $x \in B^{*}$, we define the right and left quotients of $S$ by $x$ as follows

$$
S x^{-1}=\left\{y \in B^{*} \mid y x \in S\right\} \quad \text { and } \quad x^{-1} S=\left\{y \in B^{*} \mid x y \in S\right\}
$$


We claim that

$$
\beta^{-1}\left(K_{0} b K_{1}\right)=\bigcup_{\left\{a \mid \beta(a)=x_{0} b x_{1}\right\}} \beta^{-1}\left(K_{0} x_{0}^{-1}\right) a \beta^{-1}\left(x_{1}^{-1} K_{1}\right)
$$

Indeed, if $\beta(a)=x_{0} b x_{1}$, then

$$
\beta\left(\beta^{-1}\left(K_{0} x_{0}^{-1}\right) a \beta^{-1}\left(x_{1}^{-1} K_{1}\right)\right) \subset\left(K_{0} x_{0}^{-1}\right) x_{0} b x_{1}\left(x_{1}^{-1} K_{1}\right) \subset K_{0} b K_{1}
$$

In the opposite direction, take $u \in \beta^{-1}\left(K_{0} b K_{1}\right)$. Then $\beta(u) \in K_{0} b K_{1}$ and there exists a factorization $\beta(u)=k_{0} b k_{1}$ with $k_{0} \in K_{0}$ and $k_{1} \in K_{1}$. Therefore, there is a factorization $u=u_{0} a u_{1}$ such that $\beta(a)=x_{0} b x_{1}, \beta\left(u_{0}\right)=k_{0} x_{0}^{-1}$ and $\beta\left(u_{1}\right)=x_{1}^{-1} k_{1}$. It follows that $u \in \beta^{-1}\left(K_{0} x_{0}^{-1}\right) a \beta^{-1}\left(x_{1}^{-1} K_{1}\right)$, proving the claim. Notice that formula (4.3) gives an expression of $\beta^{-1}\left(K_{0} b K_{1}\right)$ as a finite union of languages since any rational language has only a finite number of right (resp. left) quotients. Since a variety of languages is closed under left and right quotients, we have $K_{0} x_{0}^{-1}, x_{1}^{-1} K_{1} \in \mathcal{V}\left(B^{*}\right)$ and thus $\beta^{-1}\left(K_{0} x_{0}^{-1}\right), \beta^{-1}\left(x_{1}^{-1} K_{1}\right) \in \mathcal{V}\left(A^{*}\right)$. Furthermore, each product $P=\beta^{-1}\left(K_{0} x_{0}^{-1}\right) a \beta^{-1}\left(x_{1}^{-1} K_{1}\right)$ is bideterministic. Indeed, if for instance the product $P$ was not deterministic, then there would be a word $u \in P$ with a factorization of the form $u=u_{0} a u^{\prime} a u_{1}$ with $u_{0} a, u_{0} a u^{\prime} a \in \beta^{-1}\left(K_{0} x_{0}^{-1}\right) a$ and $u^{\prime} a u_{1}, u_{1} \in \beta^{-1}\left(x_{1}^{-1} K_{1}\right)$. Then $\beta(u)$ would be a word of $K_{0} b K_{1}$ with two distinct prefixes in $K_{0} b$, a contradiction. Therefore, $P \in \mathcal{C}\left(A^{*}\right)$ and $\beta^{-1}\left(K_{0} b K_{1}\right) \in \mathcal{C}\left(A^{*}\right)$, concluding the proof.

With the previous notations, we denote by $\overline{\mathbf{V}}$ the smallest variety containing $\mathbf{V}$ and closed under the operation $M \rightarrow \widehat{M}$. This variety is in general larger than $\widehat{\mathbf{V}}$ since $\widehat{\mathbf{V}}$ is not necessarily closed under the operation $M \rightarrow \widehat{M}$. On the other hand, if $\mathbf{V}=\widehat{\mathbf{V}}$, then $\mathbf{V}=\overline{\mathbf{V}}$. Let $\overline{\mathcal{V}}$ be the corresponding variety of languages. A set $\mathcal{S}$ of languages of $A^{*}$ is said to be closed under bideterministic product if, for every bideterministic product $L_{0} a L_{1}$, with $L_{0}, L_{1} \in \mathcal{S}$, one has $L_{0} a L_{1} \in \mathcal{S}$.

Theorem 4.4 For each alphabet $A, \overline{\mathcal{V}}\left(A^{*}\right)$ is the smallest boolean algebra containing $\mathcal{V}\left(A^{*}\right)$ and closed under bideterministic product.

Corollary 4.5 Let $\mathbf{V}$ be a variety of monoids and let $\mathcal{V}$ be the corresponding variety of languages. Then $\mathcal{V}$ is closed under bideterministic product if and only if $\mathbf{V}=\widehat{\mathbf{V}}$.

The next results give an explicit computation of $\overline{\mathbf{V}}$ for various varieties $\mathbf{V}$.

Proposition 4.6 The following varieties of monoids $\mathbf{V}$ satisfy $\mathbf{V}=\overline{\mathbf{V}}$ : any variety of groups, aperiodic monoids, $\mathcal{R}$-trivial monoids, $\mathcal{L}$-trivial monoids, $\mathcal{J}$-trivial monoids, monoids whose regular $\mathcal{D}$-classes are groups (respectively semigroups, idempotent semigroups, etc.), monoids whose idempotents form a submonoid, monoids with commuting idempotents and all the possible intersections of these varieties. In particular, the corresponding varieties of languages are closed under bideterministic product.

Proof. Example 2.2 shows that any variety of groups $\mathbf{V}$ satisfies $\mathbf{V}=\overline{\mathbf{V}}$. By Theorem 2.6, the expansion $\widehat{M}$ preserves regular elements and idempotents. This proves that $\mathbf{V}=\overline{\mathbf{V}}$ for the variety of monoids whose regular $\mathcal{D}$-classes are groups (respectively semigroups, idempotent semigroups, etc.), for the variety of monoids whose idempotents form a submonoid and for the variety of monoids with commuting idempotents. Furthermore it is well known (see [10] for instance) that a monoid is aperiodic (resp. $\mathcal{R}$-trivial, $\mathcal{L}$-trivial, $\mathcal{J}$-trivial) if and only if its regular $\mathcal{H}$-classes (resp. $\mathcal{R}$-classes, $\mathcal{L}$-classes, $\mathcal{J}$-classes) are trivial. Thus $\mathbf{V}=\overline{\mathbf{V}}$ also holds for these varieties. 
The proofs of the next theorems share a few standard decomposition techniques (see [1] for instance) which we recall below.

Proposition 4.7 Let $M$ be a monoid and let $I$ be its minimal ideal. Let $M_{\mathrm{r}}$ (resp. $M_{\ell}$ ) be the transformation monoid defined by the right (resp. left) action of $M$ on $I$. Then $M$ is a submonoid of $(M / I) \times M_{\mathrm{r}} \times M_{\ell}$. Furthermore, if $I$ is $\mathcal{R}$-trivial (resp. $\mathcal{L}$-trivial), then $M$ is a submonoid of $(M / I) \times M_{\ell}\left(\operatorname{resp} .(M / I) \times M_{\mathrm{r}}\right)$.

Corollary 4.8 If the minimal ideal $G$ of a monoid $M$ is a group, then $M$ is a submonoid of $(M / G) \times G$.

Proposition 4.9 Suppose that a monoid $M$ has a zero and a unique 0-minimal ideal $J$. If $J \backslash\{0\}$ is a subsemigroup of $M$, then $M \backslash\{0\}$ is a submonoid of $M$ and $M$ is a quotient of $(M \backslash\{0\}) \times U_{1}$.

Proof. Let $I=J \backslash\{0\}$. Let $x, y \in M \backslash\{0\}$ and let $s \in I$. Since $J$ is the unique 0minimal ideal of $M, I$ is contained in the ideal generated by $x$ (resp. $y$ ). In particular, $s=x_{1} x x_{2}=y_{1} y y_{2}$ for some $x_{1}, x_{2}, y_{1}, y_{2} \in M$. Since $I$ is a simple semigroup, it follows that $s^{2}=s x_{1} x x_{2} \in I$ whence $s x_{1} x \mathcal{R} s$ and $s x_{1} x \in I$. Similarly, $y y_{2} s \in I$ and thus $s x_{1} x y y_{2} s \in I$. In particular, $x y \neq 0$. Therefore, $M \backslash\{0\}$ is a submonoid of $M$.

Let $\pi:(M \backslash\{0\}) \times U_{1} \rightarrow M$ be the map defined, for every $m \in M \backslash\{0\}$, by $\pi(m, 1)=m$ and $\pi(m, 0)=0$. Then $\pi$ is a surjective morphism and thus $M$ is a quotient $(M \backslash\{0\}) \times U_{1}$.

Proposition 4.10 Suppose that a monoid $M$ has a zero and two distinct 0-minimal ideals $J_{1}$ and $J_{2}$. Then $M$ is a submonoid of $\left(M / J_{1}\right) \times\left(M / J_{2}\right)$.

Proof. Let $\pi_{1}: M \rightarrow M / J_{1}$ and $\pi_{2}: M \rightarrow M / J_{2}$ be the natural morphisms. Let $\pi: M \rightarrow\left(M / J_{1}\right) \times\left(M / J_{2}\right)$ be the morphism defined by $\pi(m)=\left(\pi_{1}(m), \pi_{2}(m)\right)$. We claim that $\pi$ is injective. Indeed, suppose that $\pi(m)=\pi\left(m^{\prime}\right)$. Then $\pi_{1}(m)=\pi_{1}\left(m^{\prime}\right)$ and $\pi_{2}(m)=\pi_{2}\left(m^{\prime}\right)$ and thus $m$ and $m^{\prime}$ are either simultaneously in $J_{1}$ (resp. $J_{2}$ ) or simultaneously in $M \backslash J_{1}$ (resp. $M \backslash J_{2}$ ). In the latter case, we have $m=m^{\prime}$. Thus we may assume that $m$ and $m^{\prime}$ belong to $J_{1}$ and to $J_{2}$. But the intersection of two distinct 0-minimal ideals is equal to $\{0\}$ and thus $m=m^{\prime}=0$. This proves the claim and the proposition.

Theorem 4.11 If $\mathbf{S l}$ is the variety of idempotent and commutative monoids, then $\overline{\mathbf{S l}}$ is the variety of $\mathcal{J}$-trivial monoids with commuting idempotents.

Proof. By Proposition 4.6, the variety $\mathbf{V}$ of $\mathcal{J}$-trivial monoids with commuting idempotents satisfies $\mathbf{V}=\overline{\mathbf{V}}$. Since $\mathbf{S l} \subset \mathbf{V}$, it follows that $\overline{\mathbf{S l}} \subset \mathbf{V}$.

Conversely, let $M \in \mathbf{V}$. We show by induction on $\operatorname{Card}(M)$ that $M \in \overline{\mathbf{S l}}$. The result is clear if $\operatorname{Card}(M)=1$, so we may assume $\operatorname{Card}(M)>1$. Since $M$ is $\mathcal{J}$ trivial, $M$ has a zero. If $M$ has (at least) two 0 -minimal ideals $J_{1}$ and $J_{2}$, then $M$ divides $\left(M / J_{1}\right) \times\left(M / J_{2}\right)$ by Proposition 4.10. Now since $\operatorname{Card}\left(M / J_{1}\right)<\operatorname{Card}(M)$ and $\operatorname{Card}\left(M / J_{2}\right)<\operatorname{Card}(M)$, it follows that $M / J_{1}, M / J_{2} \in \overline{\mathbf{S l}}$ by the induction hypothesis, whence $M \in \overline{\mathbf{S l}}$.

Assume now that $M$ has a unique 0 -minimal ideal $J$, and put $J=\{s, 0\}$. If $s$ is regular, then by Proposition 4.9, $M$ divides $(M / J) \times U_{1}$. Since $U_{1} \in \mathbf{S l}$ and $\operatorname{Card}(M / J)<\operatorname{Card}(M)$, it follows that $M / J \in \overline{\mathbf{S l}}$ by the induction hypothesis, and thus $M \in \overline{\mathbf{S l}}$. Finally suppose that $s^{2}=0$, so that $J^{2}=0$. Then by Proposition 3.1 and Theorem $3.5, M$ is a quotient of $\widehat{M / J}$. But $M / J \in \overline{\mathbf{S l}}$ by the induction hypothesis, and thus $M \in \overline{\mathbf{S l}}$. 
Theorem 4.11 can be extended as follows :

Theorem 4.12 Let $\mathbf{H}$ be a variety of groups and let $\mathbf{S l}(\mathbf{H})$ be the variety of monoids which are semilattices of groups of the variety $\mathbf{H}$. Then $\overline{\mathbf{S l}(\mathbf{H})}$ is the variety of monoids with commuting idempotents whose regular $\mathcal{J}$-classes are groups of the variety $\mathbf{H}$.

Proof. By prop 4.6, the variety $\mathbf{W}$ of all monoids with commuting idempotents whose regular $\mathcal{J}$-classes are groups of $\mathbf{H}$ satisfies $\mathbf{W}=\overline{\mathbf{W}}$. Since $\mathbf{S l}(\mathbf{H}) \subset \mathbf{W}$, it follows that $\overline{\mathbf{S l}(\mathbf{H})} \subset \mathbf{W}$.

Conversely, let $M$ be a monoid in $\mathbf{W}$. We show by induction on $\operatorname{Card}(M)$ that $M \in \overline{\mathbf{S l}(\mathbf{H})}$. The result is clear if $\operatorname{Card}(M)=1$, so we may assume $\operatorname{Card}(M)>1$. Since $M \in \mathbf{W}$, the minimal ideal of $M$ is a group $G$, and by Corollary 4.8, $M$ divides $(M / G) \times G$. But $G \in \mathbf{S l}(\mathbf{H})$, and thus it suffices to show that $M / G \in$ $\overline{\mathbf{S l}(\mathbf{H})}$. In other words, we may assume that $M$ has a zero. If $M$ has (at least) two 0-minimal ideals $J_{1}$ and $J_{2}$, then $M$ divides $\left(M / J_{1}\right) \times\left(M / J_{2}\right)$ by Proposition 4.10. Since $\operatorname{Card}\left(M / J_{1}\right)<\operatorname{Card}(M)$ and $\operatorname{Card}\left(M / J_{2}\right)<\operatorname{Card}(M)$, it follows that $M / J_{1}, M / J_{2} \in \overline{\mathbf{S l}(\mathbf{H})}$ by the induction hypothesis, whence $M \in \overline{\mathbf{S l}(\mathbf{H})}$.

Assume now that $M$ has a unique 0 -minimal ideal $J$, and put $I=J \backslash\{0\}$. If $I$ is regular (and thus, is a group), then $M$ divides $(M / J) \times U_{1}$ by Proposition 4.9 . Since $U_{1} \in \mathbf{S l}$ and $\operatorname{Card}(M / J)<\operatorname{Card}(M)$, it follows that $M / J \in \overline{\mathbf{S l}(\mathbf{H})}$ by the induction hypothesis, and thus $M \in \overline{\mathbf{S l}(\mathbf{H})}$. Finally suppose that $I$ is not regular, that is, $J^{2}=0$. Then by Theorem $3.5, M$ is a quotient of $\widehat{M / J}$. But $M / J \in \overline{\mathbf{S l}(\mathbf{H})}$ by the induction hypothesis, and thus $M \in \overline{\mathbf{S l}(\mathbf{H})}$.

Corollary 4.13 Let $\mathbf{V}$ be the variety of $\mathcal{J}$-trivial monoids with commuting idempotents and let $\mathcal{V}$ be the corresponding variety of languages. Then, for every alphabet $A, \mathcal{V}\left(A^{*}\right)$ is the smallest boolean algebra containing the languages of the form $B^{*}$, where $B$ is a subset of $A$ and closed under bideterministic product.

Proof. It is well known (see $[6,7,10]$ for instance) that, for every alphabet $A$, the class of languages of $A^{*}$ recognized by an idempotent and commutative monoid is the boolean algebra generated by the languages of the form $B^{*}$, where $B$ is a subset of $A$. The result now follows from Theorems 4.4 and 4.11 .

Corollary 4.14 Let $\mathbf{H}$ be a variety of groups. Let $\mathbf{V}$ be the variety of monoids with commuting idempotents whose regular $\mathcal{J}$-classes are groups of the variety $\mathbf{H}$. Then, for every alphabet $A, \mathcal{V}\left(A^{*}\right)$ is the smallest boolean algebra closed under bideterministic product containing the languages $B^{*}$, where $B$ is a subset of $A$ and the languages whose syntactic monoid is a group of the variety $\mathbf{H}$.

Proof. The variety $\mathbf{V}$ of monoids which are semilattices of groups of the variety $\mathbf{H}$ is equal to $\mathbf{S l} \vee \mathbf{H}$, the join of the varieties $\mathbf{H}$ and $\mathbf{S l}$. Let $\mathcal{V}$ be the corresponding variety of languages. Then for every alphabet $A, \mathcal{V}\left(A^{*}\right)$ is the boolean algebra generated by the languages whose syntactic monoid is a group of the variety $\mathbf{H}$ and by the languages of the form $B^{*}$, where $B$ is a subset of $A$. The result now follows from Theorems 4.4 and 4.11 .

Corollaries 4.13 and 4.14 should be compared with the following results of [3].

Theorem 4.15 Let $L \in A^{*}$ be a recognizable language and let $M$ be its syntactic monoid. The following conditions are equivalent:

(1) $M$ is $\mathcal{J}$-trivial with commuting idempotents, 
(2) $L$ is a boolean combination of languages of the form $A_{0}^{*} a_{1} A_{1}^{*} a_{2} \cdots a_{k} A_{k}^{*}$, where $k \geq 0, a_{1}, \ldots, a_{k} \in A, A_{0}, \ldots, A_{k} \subset A$, and, for $1 \leq i \leq k, a_{i} \notin A_{i-1} \cup A_{i}$.

Theorem 4.16 Let $\mathbf{H}$ be a variety of groups. Let $L \in A^{*}$ be a recognizable language and let $M$ be its syntactic monoid. The following conditions are equivalent:

(1) $M$ is a monoid with commuting idempotents whose regular $\mathcal{J}$-classes are groups of the variety $\mathbf{H}$,

(2) $L$ is a boolean combination of languages of the form $L_{0} a_{1} L_{1} a_{2} \cdots a_{k} L_{k}$, where $k \geq 0, a_{1}, \ldots, a_{k} \in A$, and $L_{i} \subset A_{i}^{*}$, where $A_{i}$ is a subset of $A$ such that $a_{i} \notin A_{i-1} \cup A_{i}$ and the syntactic monoid of $L_{i}$ in $A_{i}^{*}$ is a group of the variety H.

Our next result gives a description of the variety $\overline{\mathbf{V}}$ when $\mathbf{V}$ is the variety of $\mathcal{R}$-trivial and idempotent monoids (also called left regular bands). The variety $\overline{\mathbf{V}}$ is given by three equations. Two of the three equations of this description, namely

$$
(1) \quad(x y)^{\omega}=(x y)^{\omega} x \text { and } \quad(2) \quad x^{\omega} y^{\omega} x=x^{\omega} y^{\omega}
$$

characterize the $\mathcal{R}$-trivial monoids in which idempotents form a submonoid. Indeed, let $M$ be an $\mathcal{R}$-trivial monoid in which idempotents form a submonoid, and let $x, y \in M$. Then $(x y)^{\omega} \mathcal{R}(x y)^{\omega} x$ and thus $(x y)^{\omega}=(x y)^{\omega} x$. Similarly, since $x^{\omega} y^{\omega}$ is idempotent, $x^{\omega} y^{\omega} x \mathcal{R} x^{\omega} y^{\omega}$ and thus $x^{\omega} y^{\omega} x=x^{\omega} y^{\omega}$. Conversely, if $M$ satisfies the equations (1) and (2), $M$ is $\mathcal{R}$-trivial (see $[6,10]$ for instance) and the product of two idempotents is idempotent since, by (2), $x^{\omega} y^{\omega} x^{\omega} y^{\omega}=x^{\omega} y^{\omega} y^{\omega}=x^{\omega} y^{\omega}$.

The third equation of the description is $x(x y)^{\omega}=(x y)^{\omega}$ and is not a consequence of the two first equations.

Theorem 4.17 If $\mathbf{V}$ is the variety of $\mathcal{R}$-trivial and idempotent monoids, then $\overline{\mathbf{V}}$ is the variety defined by the equations $x(x y)^{\omega}=(x y)^{\omega}=(x y)^{\omega} x$ and $x^{\omega} y^{\omega} x=x^{\omega} y^{\omega}$.

Proof. Let $\mathbf{W}$ be the variety defined by the equations $x(x y)^{\omega}=(x y)^{\omega}=(x y)^{\omega} x$ and $x^{\omega} y^{\omega} x=x^{\omega} y^{\omega}$. We first show that $\hat{\mathbf{W}}=\mathbf{W}$. Let $M \in \mathbf{W}$ and let $\pi: \hat{M} \rightarrow M$ be an expansion of $M$. Since $M \in \mathbf{W}, M$ is $\mathcal{R}$-trivial and the idempotents of $M$ form a submonoid and by Proposition 4.6, the same properties hold in $\hat{M}$. In particular, $\hat{M}$ satisfies the equations $(x y)^{\omega}=(x y)^{\omega} x$ and $x^{\omega} y^{\omega} x=x^{\omega} y^{\omega}$. It follows that, for every $x, y \in \hat{M}$,

$$
(x y)^{\omega}=(x y)^{\omega} x=\left((x y)^{\omega} x\right)^{2}=(x y)^{\omega} x(x y)^{\omega} x=(x y)^{\omega} x(x y)^{\omega}
$$

In particular, $x(x y)^{\omega} \mathcal{L}(x y)^{\omega}$ and thus $(x y)^{\omega}$ and $x(x y)^{\omega}$ are regular elements of $\hat{M}$. Now, by Theorem 2.6, $\pi$ induces an isomorphism from $\langle\operatorname{Reg}(\widehat{M})\rangle$ onto $\langle\operatorname{Reg}(M)\rangle$. But since $M \in \mathbf{W}, \pi\left(x(x y)^{\omega}\right)=\pi\left((x y)^{\omega}\right)$ and thus $x(x y)^{\omega}=(x y)^{\omega}$. Thus $\hat{M} \in \mathbf{W}$ and $\hat{\mathbf{W}}=\mathbf{W}$. Since $\mathbf{V} \subset \mathbf{W}$, it follows that $\overline{\mathbf{V}} \subset \mathbf{W}$.

Conversely, let $M$ be a monoid in $\mathbf{W}$. We show by induction on $\operatorname{Card}(M)$ that $M \in \overline{\mathbf{V}}$. The result is clear if $\operatorname{Card}(M)=1$, so we may assume $\operatorname{Card}(M)>1$. Let $I$ be the minimal ideal of $M$. By Proposition 4.7, $M$ is a submonoid of $(M / I) \times M_{\ell}$. We claim that $M_{\ell} \in \mathbf{V}$. Since $M_{\ell}$ divides $M, M_{\ell}$ is $\mathcal{R}$-trivial and it suffices to show that $M_{\ell}$ is idempotent. Let $x \in M$ and let $y \in I$. Then $x y \in I$ and thus $x y$ is idempotent. It follows that $x y=(x y)^{\omega}=x(x y)^{\omega}=x x y=x^{2} y$. This shows that $x$ and $x^{2}$ have the same left action on $I$, proving the claim. Therefore it suffices to show now that $M / I \in \overline{\mathbf{V}}$. If $\operatorname{Card}(I) \geq 2$, one can conclude by induction. Otherwise, we may suppose that $I=\{0\}$.

If $M$ has (at least) two 0 -minimal ideals $J_{1}$ and $J_{2}$, then $M$ divides $\left(M / J_{1}\right) \times$ $\left(M / J_{2}\right)$ by Proposition 4.10 and we conclude by induction. Assume now that $M$ has a unique 0 -minimal ideal $J$, and put $I=J \backslash\{0\}$. If $I$ is regular, then $M$ 
divides $(M / J) \times U_{1}$ by Proposition 4.9. Since $U_{1} \in \mathbf{S l}$ and $\operatorname{Card}(M / J)<\operatorname{Card}(M)$, it follows that $M / J \in \overline{\mathbf{V}}$ by the induction hypothesis, and thus $M \in \overline{\mathbf{V}}$. Finally suppose that $I$ is not regular, that is, $J^{2}=0$. Then by Theorem $3.5, M$ is a quotient of $\overline{M / J}$. But $M / J \in \overline{\mathbf{V}}$ by the induction hypothesis, and thus $M \in \overline{\mathbf{V}}$.

Corollary 4.18 Let $\mathbf{V}$ be the variety defined by the equations $x(x y)^{\omega}=(x y)^{\omega}=$ $(x y)^{\omega} x$ and $x^{\omega} y^{\omega} x=x^{\omega} y^{\omega}$ and let $\mathcal{V}$ be the corresponding variety of languages. Then for every alphabet $A, \mathcal{V}\left(A^{*}\right)$ is the smallest boolean algebra containing the languages of the form $B^{*} a A^{*}$, where $B$ is a subset of $A$, and closed under bideterministic product.

\section{Acknowledgements}

The authors would like to thank Mário J.J. Branco for pointing out several errors in the first version of this article and Howard Straubing and the anonymous referee for useful suggestions.

\section{References}

[1] M.A. Arbib, Algebraic Theory of Machines, Languages and Semigroups, Academic Press, New-York, (1968).

[2] C.J. Ash, Finite semigroups with commuting idempotents, J. Austral. Math. Soc. (Series A) 43, (1987) 81-90.

[3] C.J. Ash, T.E. Hall and J.E. Pin, On the varieties of languages associated to some varieties of finite monoids with commuting idempotents, Information and Computation 86, (1990) 32-42.

[4] J.-C. Birget, S. Margolis and J. Rhodes. Finite semigroups whose idempotents commute or form a subsemigroup, in Semigroups and their applications, edited by S. Goberstein and P. Higgins, (Reidel, Dordrecht, Holland), (1987) 25-35.

[5] J.C. Birget, S.W. Margolis and J. Rhodes, Finite semigroups whose idempotents form a subsemigroup, Bull. Austral. Math. Soc. 41 (1990) 161-184.

[6] Eilenberg, S., Automata, Languages and Machines, Vol. A, Academic Press, New York, 1974. Vol. B, Academic Press, New York, 1976.

[7] Lallement, G., Semigroups and combinatorial applications, Wiley, New York, 1979.

[8] J.E. Pin, Variétés de langages et variétés de semigroupes, Thèse d'Etat, Paris, 1981.

[9] J.E. Pin, Propriétés syntactiques du produit non ambigu. 7th ICALP, Lecture Notes in Computer Science 85 (1980) 483-499.

[10] J.E. Pin, Variétés de langages formels, 160 p., Masson, Paris (1984). Varieties of formal languages, 138 p., North Oxford Academic (London), 1986 and Plenum (New York), 1986.

[11] J.E. Pin, H. Straubing and D. Thérien, Small varieties of finite semigroups and extensions, J. Austral. Math. Soc. 37 (1984) 269-281. 
[12] J.E. Pin, H. Straubing and D. Thérien, Locally trivial categories and unambiguous concatenation, Journal of Pure and Applied Algebra 52 (1988) 297-311.

[13] M.P. Schützenberger, 1965, On finite monoids having only trivial subgroups, Information and Control 8, 190-194.

[14] M.P. Schützenberger, Sur le produit de concaténation non ambigu, Semigroup Forum 13 (1976) 47-75.

[15] H. Straubing, Families of recognizable sets corresponding to certain varieties of finite monoids, J. Pure Appl. Algebra, 15 (1979) 305-318.

[16] H. Straubing, Aperiodic homomorphisms and the concatenation product of recognizable sets, J. Pure Appl. Algebra, 15 (1979) 319-327.

[17] P. Weil, Produits et decompositions d'automates, applications à la théorie des langages, Thèse de troisième cycle, Université Paris VII, (1985).

[18] P. Weil, Closure of varieties of languages under products with counter, JCSS 45 (1992) 316-339

J.E. Pin,

LITP, Tour 55-65

Université Paris VI et CNRS

4 Place Jussieu,

75252 Paris Cedex 05

FRANCE

e-mail:pin@litp.ibp.fr
D. Thérien,

School of Computer Science

McGill University

Montréal,

11

Québec H3A2A7

CANADA

e-mail:denis@opus.mcgill 